\title{
Three-dimensional Statistical Modeling of the Effects of the Random Distribution of Dopants in Deep Sub-micron nMOSFETs
}

\author{
E. AMIRANTE ${ }^{\mathrm{a}}$, G. IANNACCONE ${ }^{\mathrm{b}, *}$ and B. PELLEGRINI ${ }^{\mathrm{b}}$ \\ ${ }^{a}$ Lehrstuhl für Technische Elektronik, Technische Universität München, 80290 München, Germany; \\ ${ }^{\mathrm{b}}$ Dipartimento di Ingegneria dell'Informazione, Università degli studi di Pisa, Via Diotisalvi 2, I-56126 Pisa, Italy
}

\begin{abstract}
We have performed a three-dimensional statistical simulation of the threshold voltage distribution of deep submicron nMOSFETs, as a function of gate length, doping density, oxide thickness, based on a multigrid non-linear Poisson solver. We compare our results with statistical simulations presented in the literature, and show that essentially only the vertical distribution of dopants has an effect on the standard deviation of the threshold voltage.
\end{abstract}

Keywords: Statistical modeling; Dopant fluctuations; Deep submicron MOSFETs

\section{INTRODUCTION}

According to the International Technology Roadmap for Semiconductors, 1999 edition [1], the maximum allowed standard deviation $\sigma_{V T}$ of the threshold voltage of MOSFETs should scale proportionately to the supply voltage $V_{d d}$. This would imply for the present year a value of $3 \sigma_{V T}=50 \mathrm{mV}$ (minimum gate length $120 \mathrm{~nm}$ ), for $20013 \sigma_{V T}=42 \mathrm{mV}$ (minimum gate length $100 \mathrm{~nm}$ ), for $20043 \sigma_{V T}=33 \mathrm{mV}$ (minimum gate length $70 \mathrm{~nm}$ ). On the other hand, since the dispersion of $V_{T}$ is mainly due to random fluctuations of the dopant distribution in the channel, $\sigma_{V T}$ would tend to increase with increasing doping levels and decreasing channel area, associated with downscaling of CMOS technology.

It is therefore important to fully understand how the details of device parameters and of the doping profile affect the dispersion of $V_{T}$ and of the off-state current. In this paper, we address this problem by means of three-dimensional simulations on statistically meaningful ensembles of devices ( 200 devices for extracting only the average and the standard deviation, 1000 devices for obtaining the detailed distribution of $V_{T}$ ).

A few attempts at addressing the effects of the random dopant distribution from a quantitative point of view have recently appeared in the literature [2-5]. However, only Refs. [4] and [5] present a systematic statistical analysis on a three

*Corresponding author. Tel.: +39050 568677, Fax: +39050 568522, e-mail: ianna@pimac2.iet.unipi.it 
dimensional grid, limited to threshold voltage dispersion. Given the large concentrations of dopants and carriers in deep submicron MOSFETs, our model includes Fermi-Dirac statistics of electrons and holes, and incomplete ionization of dopants. In addition, it includes the effects of a polysilicon gate - recently treated in detail in [6] - and dopant fluctuations in the polysilicon gate, source and drain.

\section{MODEL AND RESULTS}

A Monte Carlo procedure is used to generate the discrete distribution of dopants in the active area for each considered device. Let us call $N_{A i}$ and $V_{i}$ the nominal acceptor doping and the volume of the Voronoi cell associated to the $i$-th gridpoint, respectively (we consider nMOSFETs). The $i$-th cell will contain on the average $\left\langle M_{i}\right\rangle=N_{A i} V_{i}$ dopants. Given the nature of the ion implantation process, the actual number of acceptors in the cell $\tilde{M}_{i}$ is randomly chosen from a Poisson distribution with average $N_{A i} V_{i}$. Then the actual doping of the $i$-th cell is $\tilde{N}_{A i}=\tilde{M}_{i} / V_{i}$. The mesh is chosen in such a way that in the channel region $N_{A i} V_{i} \ll 1$, so that, in practice, at most one dopant atom is contained in each cell.

The non-linear Poisson equation is then solved with a multi-grid method in the three-dimensional domain, considering grounded drain and source, and a voltage $V_{g}$ applied to the gate electrode:

$$
\nabla[\varepsilon(\mathbf{r}) \nabla \phi(\mathbf{r})]=-q\left[p(\mathbf{r})-n(\mathbf{r}) N_{D}^{+}(\mathbf{r})-N_{A}^{-}(\mathbf{r})\right],
$$

where $N_{D}^{+}$and $N_{A}^{-}$are the concentrations of ionized donors and acceptors, respectively, $p$ and $n$ are the semiclassical hole and electron densities computed with the Fermi-Dirac distribution [6], $\phi$ is the electrostatic potential.

Once the carrier densities are known, we solve the continuity equation for a very small drainsource voltage $V_{d s}$, in order to compute the conductance of the channel. We actually assume that carrier densities at inversion and quasiinversion do not change to first order in $V_{d s}$, so that we obtain a simplified continuity equation with only the drift term, like in Ref. [4]. The mobility is considered constant in the channel.

From the curve of the channel conductance as a function of the gate voltage, we obtain the threshold voltage $V_{T}$, as the intercept with the horizontal axis of the line extrapolating the curve in the inversion region. We have verified that the slope of such a line for a given gate voltage is independent of the detailed doping distribution: therefore, for each device, we can simply compute the channel conductance for a single value of $V_{g}$ sufficiently larger than $V_{T}$ and, once the slope of the line is known, directly extract the threshold voltage.

First, we consider a n-MOSFET with effective channel length $L$ and width $W$ of $100 \mathrm{~nm}$, gate oxide thickness $t_{o x}=3 \mathrm{~nm}$, uniform acceptor doping $N_{A}=10^{18} \mathrm{~cm}^{-3}$ in the substrate, $\mathrm{n}^{+}$poly gate $\left(N_{D g}=10^{20} \mathrm{~cm}^{-3}\right)$, drain and source junctions with a depth of $50 \mathrm{~nm}$ and donor density $N_{D}=10^{19} \mathrm{~cm}^{-3}$.

In Figure 1 the conduction band edge in the channel, on the plane at the interface between silicon and silicon oxide, is plotted for three

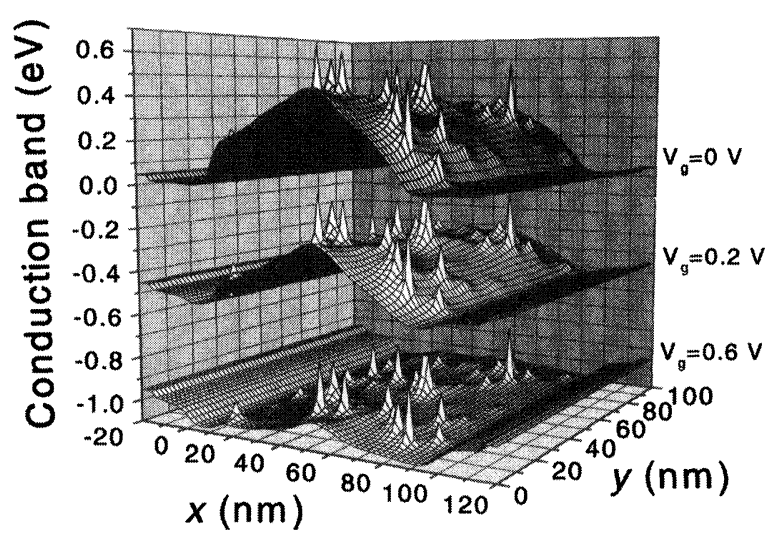

FIGURE 1 Conduction band edge in the channel, on the plane at the interface between silicon and silicon oxide, for three different gate voltage: $V_{g}=0,0.2,0.6 \mathrm{~V}$ (surfaces are translated vertically to avoid overlap). Current flows along the $x$-axis, drain and source junctions are located at $x=0$ and $x=100 \mathrm{~nm}$, respectively. 
different gate voltages: $V_{g}=0,0.2$ and $0.6 \mathrm{~V}$. Current flows along the $x$-axis, and drain and source junctions are located at $x=0$ and $x=100 \mathrm{~nm}$, respectively. The peaks are associated to the presence of individual impurities, with larger peaks due to shallower impurities, and smooth bumps due to deeper regions of higher impurity concentration.

The threshold voltage of such a device, computed in the assumption of uniform doping, is $V_{\text {Tnom }}=0.498 \mathrm{~V}$. In Figure 2, the threshold voltage frequency distribution for a sample of 1000 n-MOSFETs is shown: the average threshold voltage is $\left\langle V_{T}\right\rangle=0.486 \mathrm{~V}$, slightly smaller than $V_{\text {Tnom, }}$ and the standard deviation is $\sigma_{V T}=19 \mathrm{mV}$.

To understand the influence of the position of dopants on $V_{T}$, in Figure 3 we plot the correlation coefficient between $V_{T}$ and the total number of dopants contained in the substrate region under the gate, for $0 \leq z \leq d$ ( $z$ is the vertical direction, and $z=0$ corresponds to the $\mathrm{Si}_{-} \mathrm{SiO}_{2}$ interface). As can be seen, the correlation coefficient is close to 0.9 , for $d$ between 14 and $28 \mathrm{~nm}$; then, it decreases monotonically for larger values of $d$. These results confirm those found, on a smaller ensemble of devices, in Ref. [8].

In Figure 3 we also plot the correlation coefficient between $V_{T}$ and the number of impurities contained in a slice with $d \leq z \leq d+4 \mathrm{~nm}$ (circles). The correlation coefficient is maximum

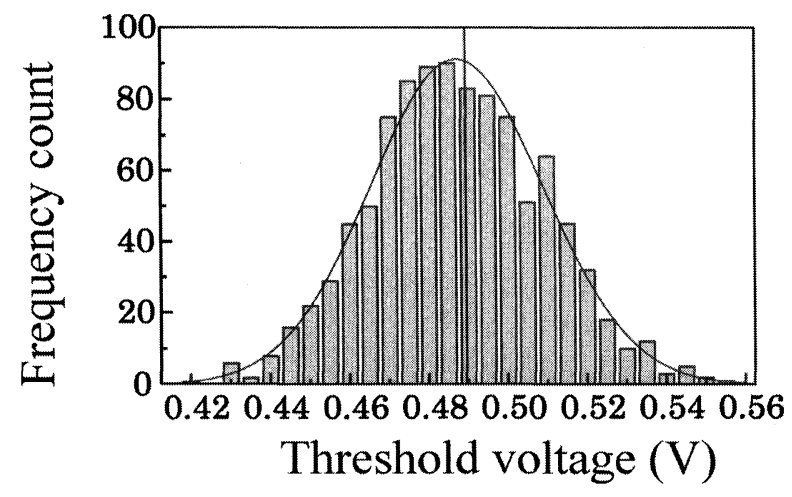

FIGURE 2 Threshold voltage frequency distribution and corresponding normal distribution for a sample of 1000 nMOSFETs with $W=L=100 \mathrm{~nm}, t_{o x}=3 \mathrm{~nm}, N_{A}=10^{18} \mathrm{~cm}^{-3}$.

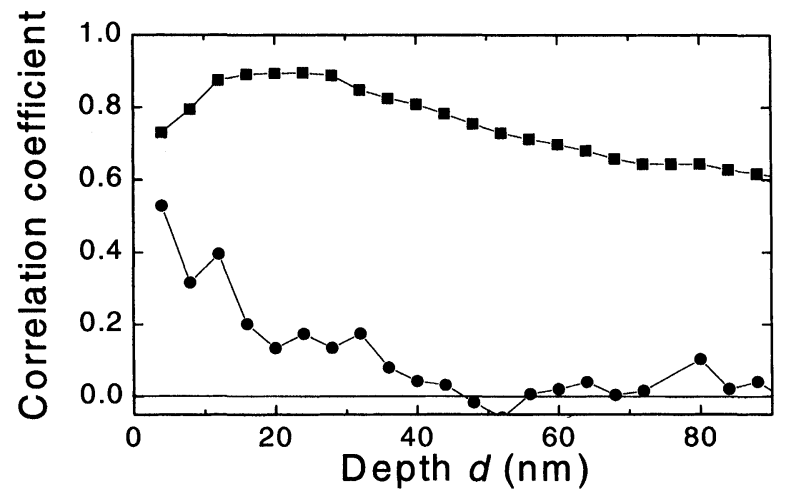

FIGURE 3 Correlation coefficient between $V_{T}$ and the total number of dopants contained in the active region at a depth smaller than $d$ (squares), and correlation coefficient between $V_{T}$ and number of impurities contained in a slice at between $d$ and $d+4 \mathrm{~nm}$ (circles).

for $d=0$ and decreases with increasing $d$ : it practically goes to zero for $d$ larger than the depletion width $W_{d}$ of the device computed with the full depletion approximation (in our case $W_{d}=34.7 \mathrm{~nm}$ ). This means that only impurities in the depletion region can influence the threshold voltage, and that shallow impurities are the most relevant for the value of $V_{T}$.

Based on an analytical derivation of the standard deviation of the threshold voltage [3], we have computed, for each device of the sample, the term $M$, defined as

$$
M=\int_{0}^{W_{d}} d z \int_{0}^{W} d y \int_{0}^{L} d x\left(1-\frac{z}{W_{d}}\right) N_{A}(x, y, z) .
$$

$M$ takes into account the total number of dopants in the depletion region, associating a larger weight to shallower impurities. In Figure 4 we show the scatter plot of $M$ versus $V_{T}$ for each of the 1000 devices in the sample. As can be seen, the correlation is extremely good, with a correlation coefficient $r=0.964$. It is worthy noticing that $M$ does not take into account the distribution of dopants in the $x$ and $y$ directions, since in (2) we simply perform an integration over $x$ and $y$. A correlation coefficient so close to 1 means that the 


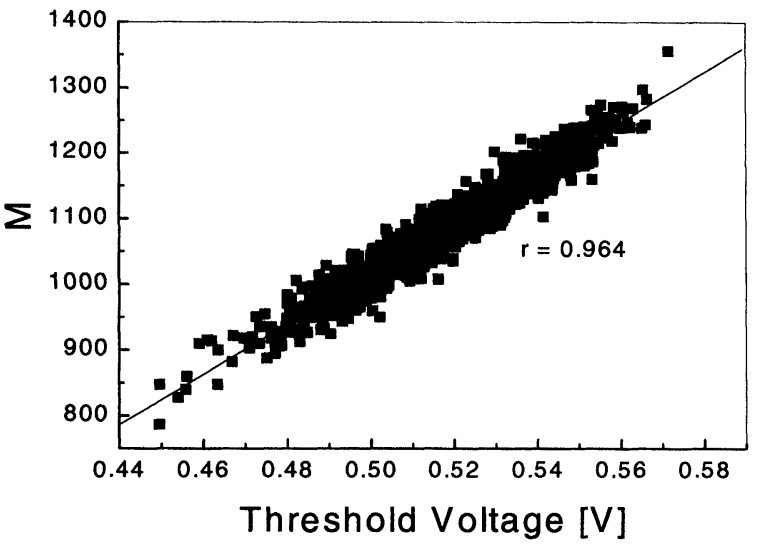

FIGURE 4 Scatter plot of $M$ defined in (2) versus $V_{T}$ for each of the 1000 devices in the sample, and least mean square fitting line. The correlation coefficient is $r=0.964$.

dispersion of $V_{T}$ depends in practice only on the vertical distribution of dopants (let us point out that the same conclusion cannot be drawn for the average of $V_{T}$ ). As a consequence, it should be possible to obtain information on the dispersion of $V_{T}$ with one-dimensional simulations, where only the vertical direction is taken into account. In such a way, the computational burden of statistical simulations could be significantly reduced. In addition, one could easily include in the simulation the effects of quantum confinement in the channel, that are prohibitive for a 3D statistical simulation, but have a significant effect on the threshold voltage of deep submicron MOSFETs.

Results from semiclassical and quantum mechanical statistical simulations in one dimension will be shown elsewhere. Here, we can anticipate that the standard deviation obtained from 1D semiclassical simulations is in very good agreement with the results from 3D simulations shown here, while the downward shift of the threshold voltage cannot be reproduced in $1 \mathrm{D}$, since it is probably due to the random occurrence of low impedance paths in the channel between drain and source, which is an inherently $3 \mathrm{D}$ phenomenon. On the other hand, the threshold voltage resulting from quantum-mechanical simulations is on average significantly larger than the semiclassical value $(130 \mathrm{mV}$ for the device structure mentioned above), while its standard deviation is only slightly (less than 10\%) larger than that obtained with the semiclassical model. This means that the actual relative dispersion of $V_{T}$ is smaller than what would appear from a semiclassical simulation.

We have repeated our three-dimensional simulations varying impurity concentration $N_{A}$ from $5 \times 10^{17}$ to $1.5 \times 10^{18} \mathrm{~cm}^{-3}$, the gate length and width from 50 to $130 \mathrm{~nm}$, and considering an oxide thickness of 2 and $3 \mathrm{~nm}$. Since the standard deviation of the error on $\left\langle V_{T}\right\rangle$ and $\sigma_{V T}$ are $\sigma_{V T} / \sqrt{N}$ and $\sigma_{V T} / \sqrt{2 N}$, respectively, where $N$ is the number of devices per sample, we have used smaller samples of 200 devices, obtaining $\sigma_{V T}$ with an accuracy of $\approx 5 \%$.

In all cases we have consistently found a downward shift of the average threshold voltage with respect to $V_{\text {Tnom: however, such a shift has }}$ been always smaller than $20 \mathrm{mV}$, with an error bar of $1-2 \mathrm{mV}$, at least factor of two smaller than that obtained in Ref. [4] with comparable parameters. The difference could be due, at least partially, to the different definition of threshold voltage used. Again, with respect to Refs. [4] and [5], we find a better agreement with results obtained from existing analytical models and simpler 1D simulations, which take into account only doping fluctuations in the vertical direction $[3,9]$.

Regarding the dependence of $\sigma_{V T}$ on the doping density, in Ref. [5] $\sigma_{V T}$ is found to be proportional to $N_{A}^{0.4}$, while from analitycal models $[3,9]$ it should be proportional to $N_{A}^{0.25}$. In Figure 5 we plot the logarithm of $\sigma_{V T}$ versus the logarithm of the doping density both for the device structure described above and for one with $t_{o x}=2 \mathrm{~nm}$ and other parameters unchanged. The slopes of the least mean squart fitting lines, i.e., the exponent of $N_{A}$, are 0.239 and 0.289 , respectively, confirming the predictions of analytical models and the results of Ref. [10]. The dependence of $\sigma_{V T}$ on the effective gate length $L$ is in good agreement with results in the literature: data in Figure 6 are obtained with $N_{A}=10^{-18} \mathrm{~cm}^{-3}, t_{o x}=3 \mathrm{~nm}$, and $W=L$, and are compared with the formula derived analytically in [3]. 


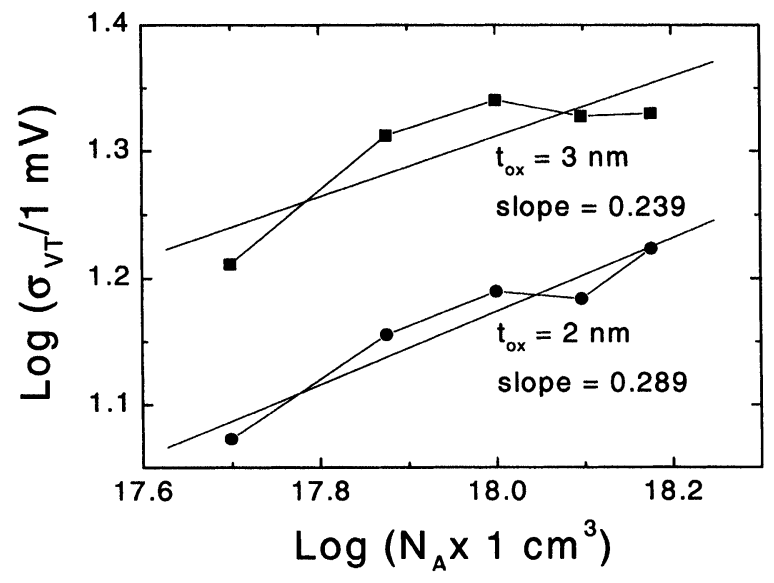

FIGURE 5 Logarithm of $\sigma_{V T}$ versus the logarithm of the doping density $N_{A}$ for a device with $L=W=100 \mathrm{~nm}$, and two different values of $t_{o x}: 3 \mathrm{~nm}$ (squares) and $2 \mathrm{~nm}$ (circles). Also plotted are the least mean square best fitting lines with related slopes.

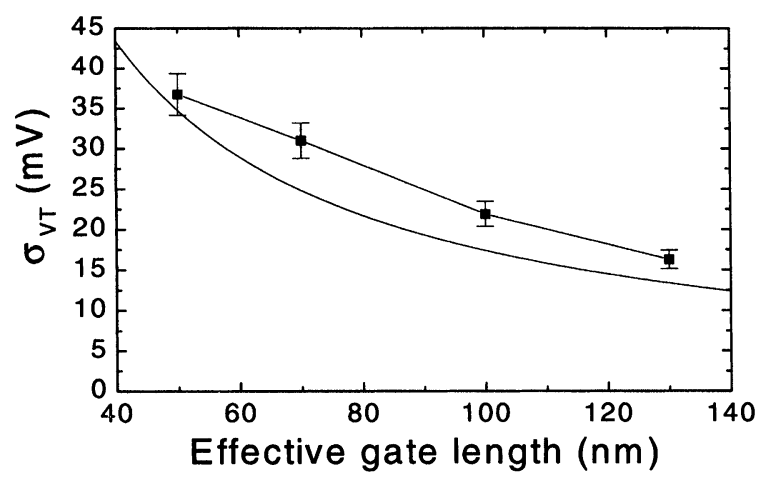

FIGURE $6 \sigma_{V T}$ as a function of the effective gatelength $L=W$, for $t_{o x}=3 \mathrm{~nm}$ and $N_{A}=10^{18} \mathrm{~cm}^{-3}$ obtained from simulations (squares) and from the analytical formula of Ref. [3].

In conclusion, we have performed a threedimensional statistical simulation of the threshold voltage distribution of deep submicron nMOSFETs, based on a multigrid non-linear
Poisson solver. We have shown that the standard deviation of $V_{T}$ essentially depends only on the vertical distribution of dopants, and that the distribution of dopants in the horizontal plane has an effect only on the average downward shift of $V_{T}$. Compared with existing results from statistical simulations $[4,5]$, we find some differences that require further analysis.

\section{References}

[1] Internation Technology Roadmap for Semiconductors1999 edition, Semiconductor Industry Association (San Jose, USA, 1999).

[2] Wong, H. S. and Taur, Y. (1993). "Three-dimensional "atomistic" simulation of discrete random dopant distribution effects in sub $-0.1 \mu \mathrm{m}$ MOSFETs", IEDM Tech. Dig., pp. 705-708.

[3] Stolk, P. A., Widdershoven, F. P. and Klaassen, D. B. M. (1998). "Modeling statistical dopant fluctuations in MOS transistors", IEEE Trans. Electron Devices, 45, 1960.

[4] Asenov, A. (1998). "Random Dopant Induced Threshold

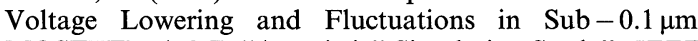
MOSFET's: A 3-D “Atomistic" Simulation Study", IEEE Trans. Electron Devices, 45, 2505.

[5] Asenov, A. and Saini, S. (1999). "Suppression of random dopant-induced threshold voltage fluctuations in sub $-0.1 \mu \mathrm{m}$ MOSFETs with epitaxial and $\delta$-doped channels", IEEE Trans. Electron Devices, 46, 1718.

[6] Asenov, A. and Saini, S. (2000). "Polysilicon gate enhancement of the random dopant induced threshold voltage fluctuations in $\mathrm{sub}-100 \mathrm{~nm}$ MOSFETs with ultrathin gate oxide", IEEE Trans. Electron Devices, 47,805 .

[7] Sze, S. (1981). Physics of semiconductor devices, New York: Wiley and Sons, 2nd edn.

[8] Vasileska, D., Gross, W. J. and Ferry, D. K. (1998). "Modeling of deep-submicrometer MOSFETs: random impurity effects, threshold voltage shifts and gate capacitance attenuation", Proc. of IWCE6 (IEEE, Piscataway) p. 259.

[9] Taur, Y. and Ning, T. H. (1998). Fundamentals of modern VLSI devices, Cambridge: Cambridge University Press.

[10] Vasileska, D., Gross, W. J. and Ferry, D. K. (2000). "Monte Carlo particle-based simulations of deep submicron n-MOSFETs with real space treatment of electron-electron interaction and electron-impurity treatment", Superlattices and Microstructures, 27, 148. 

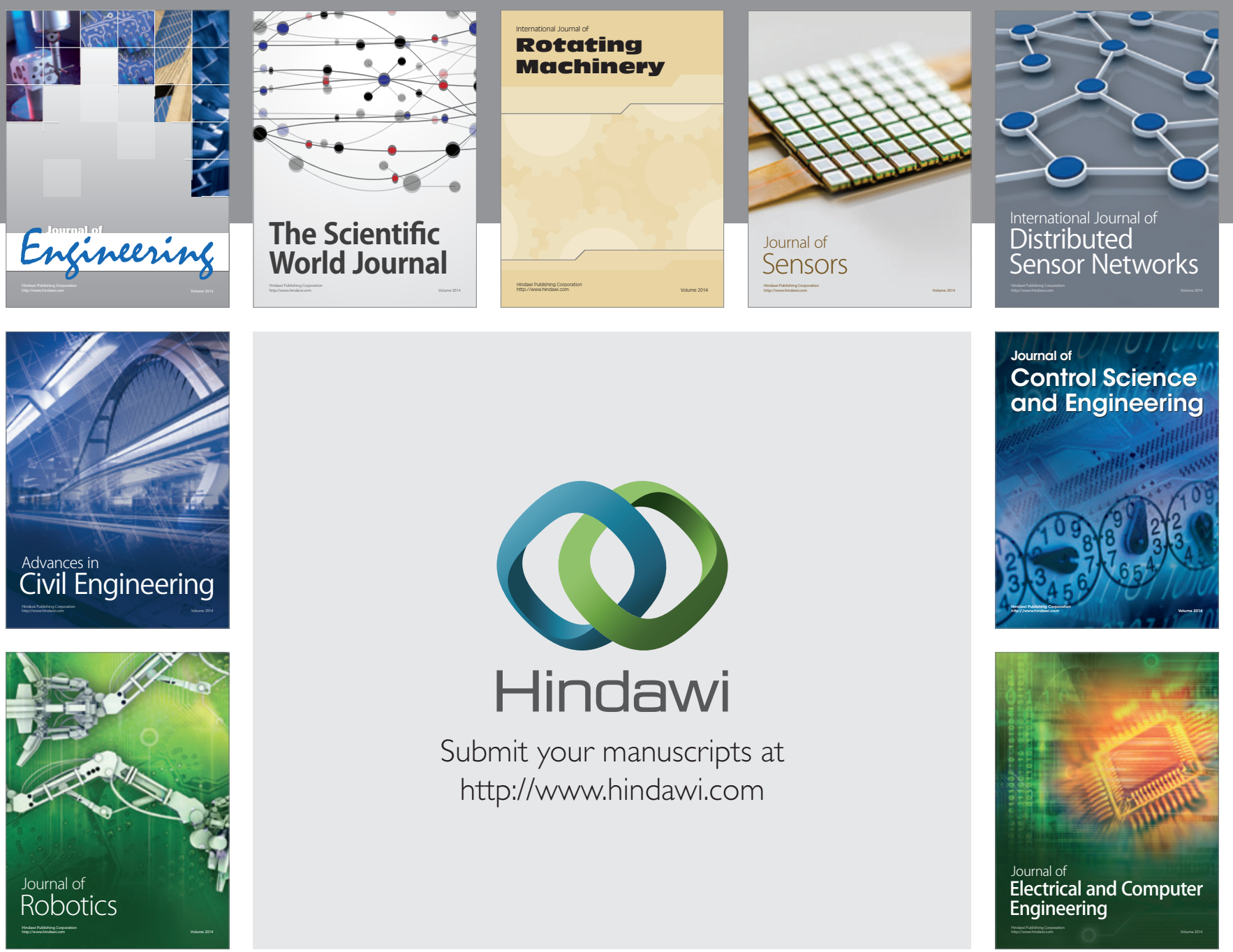

Submit your manuscripts at

http://www.hindawi.com
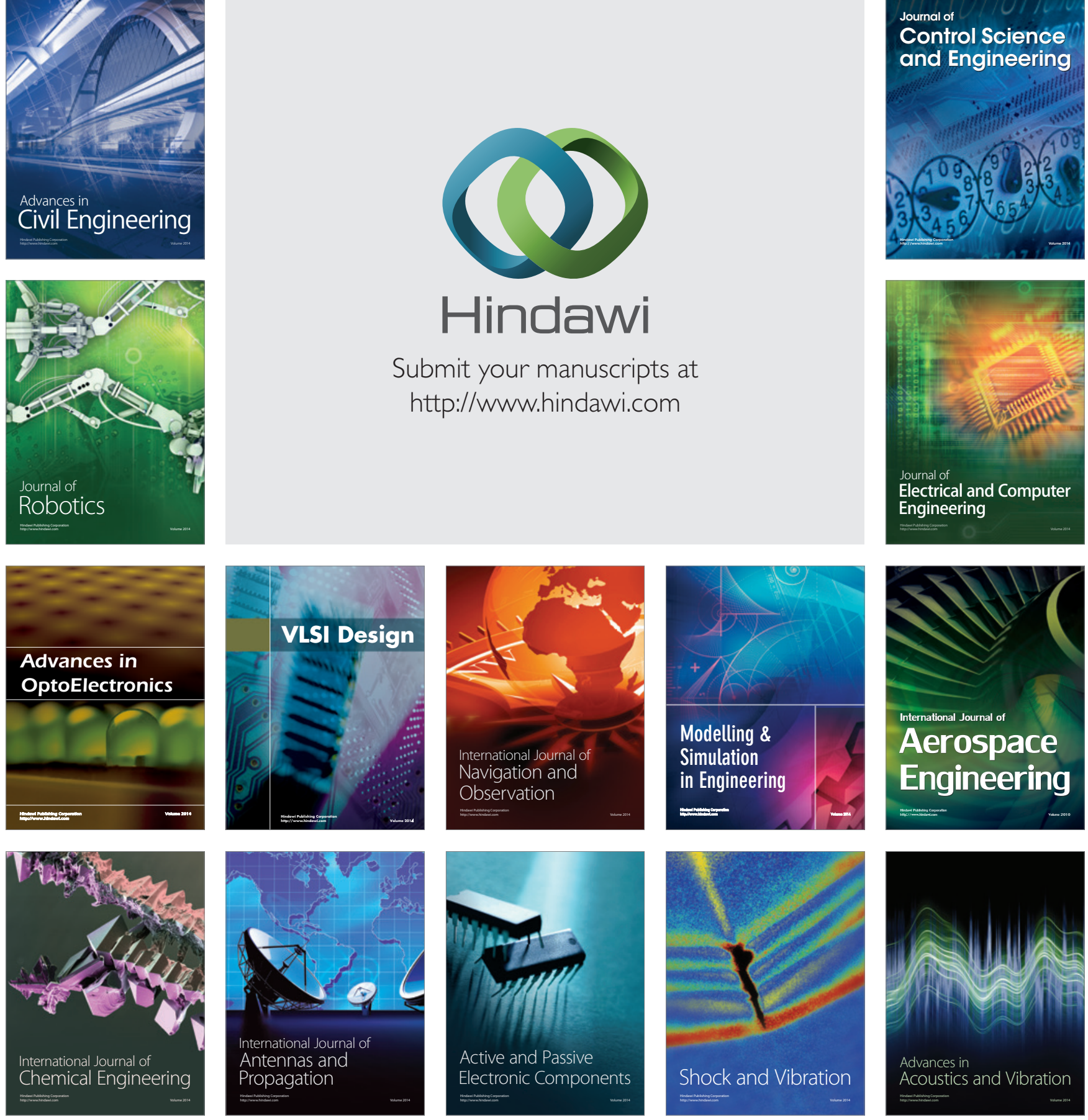